\title{
A novel input design approach for systems with quantized output data
}

\author{
Boris I. Godoy, Patricio E. Valenzuela, Cristian R. Rojas, Juan C. Agüero, and Brett Ninness
}

\begin{abstract}
In this paper, we explore the problem of input design for systems with quantized measurements. For the input design problem, we calculate and optimize a function of the Fisher Information Matrix (FIM). The calculation of the FIM is greatly simplified by using known relationships of the derivative of the likelihood function, and the auxiliary function arising from the Expectation Maximization (EM) algorithm. To optimize the FIM, we design an experiment using a recently published method based on graph theory. A numerical example shows that the proposed experiment can be successfully used in quantized systems.
\end{abstract}

\section{INTRODUCTION}

In the present paper, we explore the problem of input design for systems subject to quantized output data. This problem is of relevance because of the many applications where quantized systems are used. For example, quantized systems are widely used in network control and wireless communications, due to the restricted bandwidth of the communication channels. In fact, due to constraints on the communication channels, such as noise and limited bandwidth, it is impossible to transmit data with infinite precision. Thus, quantization is an effective way to reduce use of the transmission resource, and meet the bandwidth constraint of a communication channel. However, quantization is a lossy compression method, hence the validity or performance of parameter identification may be affected by quantization. This could then result in the deteriorated performance of adaptive control techniques.

In wireless communications, for example, quantized system identification is crucial for echo cancellation and blind communication channel estimation, see [11] and the references therein. This means that quantized system identification is very important as the use of wireless technologies is now extensive in many areas of society, and it is expected to increase.

Another type of quantized system is where the quantizer can take only 2 possible values (binary signals). This kind of configuration has attracted considerable attention. In fact,

B. I. Godoy acknowledges support given by CONICYT through its postdoctoral fellowship program 2011. This work was also supported in part by the Swedish Research Council under contracts 621-2010-5819 and 621-2011-5890, and by the European Research Council under the advanced grant LEARN, contract 267381 .

B. I. Godoy, J. C. Agüero, and B. Ninness are with the Centre for Complex Dynamic Systems \& Control, The University of Newcastle, NSW 2308, Australia, \{boris.godoy, juan.aguero,brett.ninness\} anewcastle.edu. au.

P. E. Valenzuela and C. R. Rojas are with the Automatic Control Lab and ACCESS Linnaeus Center, School of Electrical Eng., KTH-Royal Institute of Technology, SE-100 44 Stockholm, Sweden, \{pva, crro\} akth.se. binary-valued (or quantized) sensors are commonly used in practical systems. They are exemplified by switching sensors for exhaust gas oxygen, photoelectric sensors for position, Hall-effect sensors for speed and acceleration, gas content sensors in the gas and oil industry, and distributed one-bit wireless sensors. Particularly, for any remotely controlled system, signal quantization is mandatory. Compared to regular sensors, binary-valued sensors are usually far more cost effective. However, they provide very limited information on the process.

In order to improve the estimation quality of the identified quantized model, it is usually possible to manipulate the input signal to be used during the identification experiment. The input design problem has been widely studied, both in the statistics literature [8], [9], [17] and in the engineering literature [1], [10], [12], [14], [21]-[23], [32], primarily focusing on linear systems. Some extensions to nonlinear systems have been considered in [13], [15], [18], [29].

The problem of input design for quantized system has been treated in previous works [5]-[7], [30]. The idea in [30] is to establish conditions under which consistent estimates can be obtained. In particular, they recommend the use of periodic signals. Even though periodic inputs have shown to asymptotically achieve the Cramer-Rao lower bound (CRLB), their use may be inadequate for tracking control applications [19].

In [7], the input design problem is carried out by using a worst-case framework, based on the set-membership paradigm of uncertainty representation. There, the authors assume uncertainty in the parameters, and extend a previous work [6] from binary sensors to multilevel sensor thresholds.

The work [19] asserts that the input assuring strong consistency of the estimates is a persistently exciting signal of certain order $r$, as long as the quantizer is optimal in the sense of minimizing the error between the measurement and the input to the quantizer. However, the design of this signal is not provided.

In this paper, we address the input design problem for the identification of quantized systems. To this end, we consider an approach recently proposed by the authors in [28], consisting of a graph-theoretical technique leading to a convex optimization problem. The missing ingredient needed for using this approach is an estimate of the Fisher Information Matrix (FIM) obtained when using a given (periodic) input; such an estimate is proposed in this paper, based on the socalled Fisher identity [20].

In our paper, we will consider that the true system is known during the input design stage. This is a standard assumption for most optimal input design methods, and it 
is a previous and necessary step in order to perform robust experiment design [24].

The remainder of this paper is as follows: In section 2 we describe the problem. In section 3 , we recall how to estimate the system subject to quantized measurements using the Maximum likelihood (ML) estimator. In section 4, we describe our method for input design. In section 5, we describe an example showing the advantage of our method. Finally, in Section 6, we draw conclusions.

\section{Statement of The PROBlem}

We focus on the problem of input design for quantized output systems. In particular, we have a system as in Fig. 1. We consider the scheme where noise enters between the linear system and the quantizer. A block diagram for the setup is shown in Fig. 1. The noise, $w_{t}$, is assumed to be zero-mean Gaussian with variance $\sigma^{2}$. We also assume that $\sigma^{2}$ is known.

Remark 1: Note that the assumption about the noise variance being known is only necessary for the experiment design, and not for the estimation problem. As shown in [11], the noise variance can also be estimated.

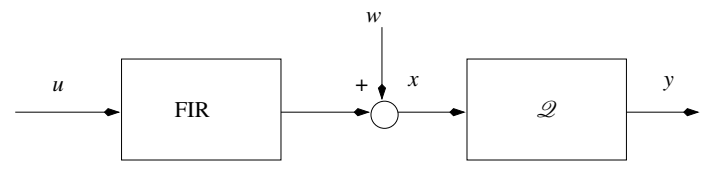

Fig. 1. FIR system with quantized output.

The system can be described by

$$
\begin{aligned}
& x_{t}=\varphi_{t}^{T} \theta+w_{t}, \quad w_{t} \sim \mathscr{N}\left(0, \sigma^{2}\right) \\
& y_{t}=\mathscr{Q}\left[x_{t}\right],
\end{aligned}
$$

where $x_{t}$ is a scalar, the parameter vector $\theta$ is of dimension $q, \varphi_{t}^{T}$ is a matrix of dimension $1 \times q$, and $\sigma^{2} \in \mathbb{R}$ is the noise variance. $\mathscr{Q}: \mathbb{R} \rightarrow \mathscr{V}$ represents a quantizer, which is a map from the set of a scalars, to the finite set $\mathscr{V}=\left\{v_{1}, v_{2}, \cdots, v_{M}\right\}$ of scalars, i.e.

$$
\mathscr{Q}[x]=\left\{\begin{array}{rl}
v_{1} & \text { if } x \in \Omega_{1} \\
\vdots & \\
v_{M} & \text { if } x \in \Omega_{M}
\end{array},\right.
$$

where $\Omega_{i}, i=1, \cdots, M$ are subsets of $\mathbb{R}$.

The problem of interest is to design the input $U_{N}:=$ $\left[\begin{array}{llll}u_{1} & u_{2} & \ldots & u_{N}\end{array}\right]^{T}$ such that the parameter $\theta$ is better estimated compared to any other input signal. To formulate the input design problem, consider the FIM

$$
\mathscr{I}_{F}=\mathbb{E}\left\{\frac{\partial l(\theta)}{\partial \theta} \frac{\partial l(\theta)}{\partial \theta^{T}}\right\} \in \mathbb{R}^{q \times q},
$$

where

$$
l(\theta)=\log p\left(Y_{N} \mid \theta\right)
$$

is the log-likelihood function, with $p\left(Y_{N} \mid \theta\right)$ being the probability distribution of the data, $Y_{N}=\left\{y_{1}, \ldots, y_{N}\right\}$, given the parameter $\theta$. The FIM defines the maximal accuracy that an unbiased estimator of $\theta, \hat{\theta}$, can achieve, due to the CramérRao bound [12]:

$$
\operatorname{cov}(\hat{\theta}) \geq \mathscr{I}_{F}^{-1}
$$

Given a matrix non-decreasing ${ }^{1}$ and convex function $h$ : $S_{+}^{q} \rightarrow \mathbb{R}$, where $S_{+}^{q}$ corresponds to the space of symmetric and positive-semidefinite matrices of dimension $q \times q$, an input design problem can be posed as

$$
\begin{array}{ll}
\min _{U_{N}} & h\left(\mathscr{I}_{F}\right) \\
\text { s.t. } & \frac{1}{N}\left\|U_{N}\right\|_{2}^{2} \leq \alpha,
\end{array}
$$

where $\alpha>0$ is an upper bound on the available input power. The input design procedure that we will describe in Section IV takes the input power constraint implicitly into account, by restricting the input to take values from a finite alphabet.

\section{BACKGROUND ON ML ESTIMATION BY USING THE EM ALGORITHM WITH QUANTIZED OUTPUT DATA}

The Maximum Likelihood (ML) estimation problem can be posed as:

$$
\hat{\theta}=\arg \max _{\theta} l(\theta)
$$

where the log-likelihood function $l(\theta)$ is given by (4). For future reference we define $X_{N}=\left\{x_{1}, \ldots, x_{N}\right\}$. For the problem of interest here, we can write (4) as

$$
l(\theta)=\sum_{t=1}^{N} l_{t}(\theta), \quad l_{t}(\theta)=\log p\left(y_{t} \mid Y_{t-1}, \theta\right) .
$$

Assumption 1: The input $U_{N}:=\left\{u_{1}, \ldots, u_{N}\right\}$ is an exogenous deterministic signal, and the FIR system is in open loop. Notice that the system (of interest) is FIR, thus, $l_{t}(\theta)=$ $\log p\left(y_{t} \mid Y_{t-1}, \theta\right)=\log p\left(y_{t} \mid \theta\right)$.

Assumption 2: The vector of parameters $\theta$, the input $\left(u_{t}\right)$ and the noise $\left(w_{t}\right)$ satisfy regularity conditions guaranteeing that the solution $\hat{\theta}$ of the optimization problem in (7) converges (in probability or a.s.) to the true solution $\theta_{0}$.

The Expectation Maximization (EM) algorithm consists of two steps: (i) the calculation of an auxiliary function (E-step) and, (ii) the optimization of this auxiliary function (M-step). The E-step is obtained by calculating function $\mathbf{Q}\left(\theta, \hat{\theta}_{i}\right)$ :

$$
\mathbf{Q}\left(\theta, \hat{\theta}_{i}\right):=\int_{\mathbb{R}^{n \times N}} \log \left[p\left(X_{N}, Y_{N} \mid \theta\right)\right] p\left(X_{N} \mid Y_{N}, \hat{\theta}_{i}\right) d X_{N}
$$

The M-step is then given by

$$
\hat{\theta}_{i+1}=\arg \max _{\theta} \mathbf{Q}\left(\theta, \hat{\theta}_{i}\right) .
$$

In [11], it was shown that under the stated assumptions, the estimate of the parameters can be found using the EM algorithm. This algorithm is summarized in the following theorem:

\footnotetext{
${ }^{1} h$ is a matrix non-decreasing function if $h(A) \leq h(B)$ whenever $A \leq B[4$, pp. 108].
} 
Theorem 1: Consider the system given in (1), Assumptions 1 and 2, and the maximization problem stated in (10), then the M-step of the EM algorithm is given by:

$$
\hat{\theta}_{i+1}=\left[\sum_{t=1}^{N} \varphi_{t} \varphi_{t}^{T}\right]^{-1} \sum_{t=1}^{N} \hat{x}_{t},
$$

where

$$
\hat{x}_{t}=\varphi_{t}^{T} \hat{\theta}_{i}+\sigma \frac{I_{t}^{(1)}}{I_{t}^{(0)}}
$$

with $I_{t}^{(0)} \in \mathbb{R}, I_{t}^{(1)} \in \mathbb{R}$ given by

$$
\begin{aligned}
I_{t}^{(0)} & =\frac{1}{\sqrt{2 \pi}} \int_{\tilde{\mathscr{S}}_{t}} \exp \left\{-\frac{1}{2} \tilde{x}_{t}^{2}\right\} d \tilde{x}_{t}, \\
I_{t}^{(1)} & =\frac{1}{\sqrt{2 \pi}} \int_{\tilde{\mathscr{S}}_{t}} \tilde{x}_{t} \exp \left\{-\frac{1}{2} \tilde{x}_{t}^{2}\right\} d \tilde{x}_{t},
\end{aligned}
$$

and $\tilde{\mathscr{S}}_{t}=\left\{\tilde{x}_{t}: \mathscr{Q}\left[\sigma \tilde{x}_{t}+\varphi_{t}^{T} \hat{\theta}_{i}\right]=y_{t}\right\}$.

Developing further the expressions for the integrals $I_{t}^{(0)}$, $I_{t}^{(1)}$, we can obtain:

$$
I_{t}^{(0)}=0.5 \operatorname{erf}(\tilde{\mathscr{S}} / \sqrt{2}), \quad I_{t}^{(1)}=-\left.\frac{e^{-x^{2} / 2}}{\sqrt{2 \pi}}\right|_{\tilde{\mathscr{S}}},
$$

where

$$
\operatorname{erf}(\mathscr{A})=\frac{2}{\sqrt{\pi}} \int_{\mathscr{A}} e^{-u^{2}} d u
$$

\section{INPUT DESIGN}

\section{A. Input design via graph theory}

In order to address the input design problem posed in Section II, we restrict the input signal to be a realization of a time-homogeneous Markov chain of finite memory length, equal to $q$, taking values from a finite alphabet $\mathscr{C}$ of cardinality $c$ [28].

Remark 2: Notice that the Markov chain (of interest in this paper) is run from a previously chosen node. This node is chosen according to the stationary distribution of the Markov chain. Thus, the Markov chain starts in "stationary" state.

Remark 3: While the requirement of stationarity is standard in input design, the constraint of being a Markov process of finite memory may seem a bit restrictive. However, as shown in [18], [28], since the quantized model has a nonlinear FIR structure (i.e., the output $y_{t}$ depends on a finite number of present and past values of $u_{t}$ ), this constraint is actually not more restrictive than the one on stationarity (if the memory length of $u_{t}$ is equal to the memory length of the system). Forcing $u_{t}$ to belong to a finite alphabet, on the other hand, is quite common in many application, e.g., in communication systems.

Under the above mentioned conditions, the input signal can be represented by its stationary probability mass function (pmf) $f\left(U_{q}\right)$. Furthermore, due to the requirement of stationarity, it has been shown in [28] that the set of valid pmf's $f\left(U_{q}\right)$ corresponds to the convex hull of pmf's describing prime cycles in a de Bruijn graph. To explain this fact in more detail, we first need some definitions.

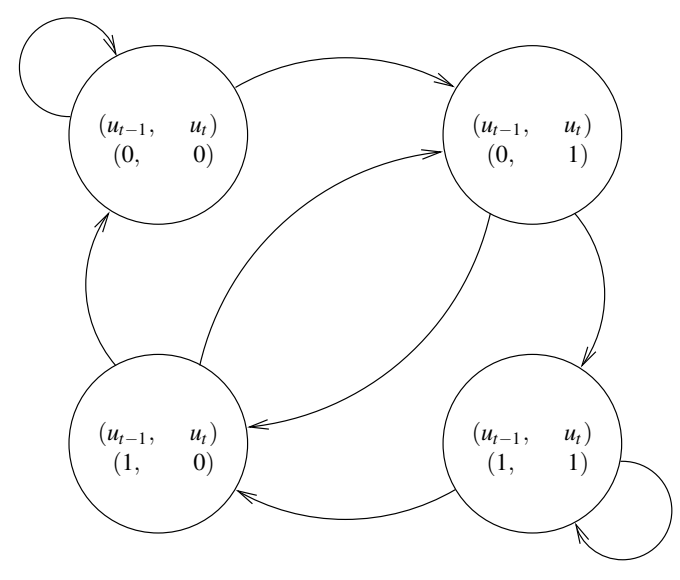

Fig. 2. de Bruijn graph derived from $\mathscr{C}^{q}$, with $c=2, q=2$, and $\mathscr{C}=\{0,1\}$.

The set $\mathscr{C}^{q}$ of possible values for $U_{q}$ is composed of $c$ elements, which can be viewed as nodes in a graph. In addition, the transitions between the elements in $\mathscr{C}^{q}$, as described by a stationary process of memory $q$, are given by the feasible values of $u_{t+q}$ when we move from $\left(u_{t}, \ldots, u_{t+q-1}\right)$ to $\left(u_{t+1}, \ldots, u_{t+q}\right)$, for $t \geq 0$. The edges between the elements in $\mathscr{C}^{q}$ denote the possible transitions between the states, represented by the nodes of the graph, called a de Bruijn graph, $\mathscr{G}_{\mathscr{C} q}$. Figure 2 illustrates this idea, when $c=2, q=2$, and $\mathscr{C}=\{0,1\}$. From this figure we can see that, if we are at node $(0,1)$ at time $t$, then we can only end at node $(1,0)$ or $(1,1)$ at time $t+1$.

A prime cycle in a de Bruijn graph is an elementary cycle ${ }^{2}$ whose set of nodes do not have a proper subset which is an elementary cycle [31, p. 678]. It can be shown that the prime cycles of this de Bruijn graph allow us to describe the set of possible stationary pmf's of memory length $q$ over $\mathscr{C}$. Specifically, for each prime cycle we can associate a pmf $f^{(i)}$ corresponding to a uniform distribution with support in the set of elements of the prime cycle [31, pp. 681]. Then, the convex hull of the $f^{(i)}$ 's corresponds to the set of stationary pmf's over the graph [31, Theorem 6].

The prime cycles of the de Bruijn graph can be derived from the elementary cycles of $\mathscr{G}_{\mathscr{C} q-1}$ [31, Lemma 4], which, in turn, can be determined using standard graph algorithms [16], [26]. As an example, consider the graph in Figure 3. One elementary cycle for this graph is $(0,1,0)$. Using Lemma 4 in [31], the elements of one prime cycle for the graph $\mathscr{G}_{\mathscr{C} 2}$ are obtained as a concatenation of the elements in the elementary cycle $(0,1,0)$, i.e., $((0,1),(1,0),(0,1))$.

The method for the design of input signals proposed in [28] can now be described as follows:

1) Compute all the elementary cycles of $\mathscr{G}_{\mathscr{C} q-1}$.

2) Compute all the prime cycles of $\mathscr{G}_{\mathscr{C} q}$ by extending the elementary cycles of $\mathscr{G}_{\mathscr{C} q-1}$.

3) Generate the input signals $\left\{u_{t}^{(i)}\right\}_{t=0}^{N_{\text {sim }}}$ by running the

\footnotetext{
${ }^{2}$ An elementary cycle is a sequence of vertices $\left(v=v_{1}, v_{2}, \ldots, v_{k}=v\right)$ such that each $\left(v_{i}, v_{i+1}\right)$ is an edge of the graph, and where no vertex but the first and last appears twice in the sequence.
} 


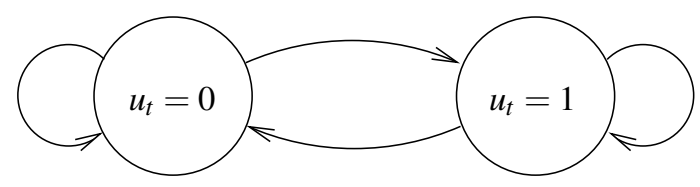

Fig. 3. de Bruijn graph derived from $\mathscr{C}^{q}$, with $c=2, q=1$, and $\mathscr{C}=\{0,1\}$.

prime cycles $\left(N_{\text {sim }}\right.$ must be sufficiently large as will be described in the next subsection).

4) Approximate the information matrix $\mathscr{I}_{F}^{(i)}$ when using $u_{t}^{(i)}$ as input. This step will be described in more detail in the next subsection.

5) Define $\gamma:=\left\{\alpha_{1}, \ldots, \alpha_{n}\right\}$, where $n$ is the number of prime cycles. Find $\gamma^{\mathrm{opt}}:=\left\{\alpha_{1}^{\mathrm{opt}}, \ldots, \alpha_{n}^{\mathrm{opt}}\right\}$ by solving:

$$
\gamma^{\mathrm{opt}}:=\arg \min _{\gamma \in \mathbb{R}^{n}} h\left(\mathscr{I}_{F}^{\mathrm{app}}(\gamma)\right)
$$

where

$$
\begin{aligned}
\mathscr{I}_{F}^{\mathrm{app}}(\gamma) & =\sum_{i=1}^{n} \alpha_{i} \mathscr{I}_{F}^{(i)}, \\
\sum_{i=1}^{n} \alpha_{i} & =1, \\
\alpha_{i} & \geq 0, \text { for all } i \in\{1, \ldots, n\} .
\end{aligned}
$$

The procedure mentioned above computes $\gamma^{\text {opt }}$ which defines the optimal pmf $f^{\text {opt }}\left(U_{q}\right)=\sum_{i=1}^{n} \alpha_{i}^{\text {opt }} f^{(i)}\left(U_{q}\right)$. Notice that $\mathscr{I}_{F}^{\text {app }}(\gamma)$ in (17) is linear in the decision variables. Therefore, the problem (16)-(19) is convex.

Finally, in order to generate the resulting input signal from the optimal $\operatorname{pmf} f^{\text {opt }}\left(U_{q}\right)$, it is necessary to find a transition probability matrix satisfying the graph constraints and having $f^{\text {opt }}$ as stationary distribution. One possible transition matrix defined on the support of $f^{\text {opt }}$, as described in [27], is:

$$
\mathbf{P}\left\{u_{t} \mid u_{t-1}, \ldots, u_{t-q+1}\right\}=\frac{f\left(u_{t-q+1}, \ldots, u_{t}\right)}{\sum_{u \in \mathscr{C}} f\left(u_{t-q+1}, \ldots, u_{t-1}, u\right)} .
$$

The input signal is initialized by picking a node of the graph at random, according to the distribution $f^{\text {opt }}$, and the rest of the sequence is simulated according to (20).

Remark 4: The computational complexity of the method described here is mainly related with the computation of elementary cycles. A time bound for the computation of elementary cycles is given by $O\left(c^{q}(c+1)\left(c_{e}+1\right)\right)$, where $c_{e}$ is the number of elementary cycles, given by [16, p. 77].

$$
c_{e}:=\sum_{i=1}^{c^{q}-1}\left(\begin{array}{c}
c^{q} \\
c^{q}-i+1
\end{array}\right)\left(c^{q}-i\right) !
$$

As expected, the number of elementary cycles grows as $c$ and $q$ increase. An approach to avoid the computation of the entire set of elementary cycles is still an open problem and topic of ongoing research.

\section{B. Calculation of the Information Matrix}

Lemma 1: For systems with quantized output data of the form (1), we have that the FIM can be approximated by the following expression:

$$
\mathscr{I}_{F} \approx \frac{2}{\pi \sigma^{2} N_{\text {sim }}} \sum_{t=1}^{N_{\text {sim }}}\left(\frac{\left.e^{-x^{2} / 2}\right|_{\tilde{\mathscr{I}}_{t}}}{\left.\operatorname{erf}(x / \sqrt{2})\right|_{\tilde{\mathscr{T}_{t}}}}\right)^{2} \varphi_{t} \varphi_{t}^{T} .
$$

Proof: We start off using the Fisher identity [20, p.80]

$$
\left.\frac{\partial l(\theta)}{\partial \theta}\right|_{\theta=\theta_{0}}=\left.\frac{\partial \mathbf{Q}\left(\theta, \theta_{0}\right)}{\partial \theta}\right|_{\theta=\theta_{0}}
$$

where $\mathbf{Q}\left(\theta, \theta_{0}\right)$ is the auxiliary function arising from the EM algorithm.

In [11], it was demonstrated that

$$
\left.\frac{\partial \mathbf{Q}\left(\theta, \theta_{0}\right)}{\partial \theta}\right|_{\theta=\theta_{0}}=\frac{1}{\sigma} \sum_{t=1}^{N} \varphi_{t} \frac{I_{t}^{(1)}}{I_{t}^{(0)}} .
$$

Following the same procedure as in [20, p.80], we can evaluate (23) for each sample $t=1, \ldots, N$ obtaining

$$
\left.\frac{\partial l_{t}(\theta)}{\partial \theta}\right|_{\theta=\theta_{0}}=\left.\frac{\partial \mathbf{Q}_{t}\left(\theta, \theta_{0}\right)}{\partial \theta}\right|_{\theta=\theta_{0}},
$$

where $l_{t}$ is defined in (8), and $\mathbf{Q}_{t}\left(\theta, \theta_{0}\right)$ can be written as

$$
\mathbf{Q}_{t}\left(\boldsymbol{\theta}, \theta_{0}\right)=\int_{\tilde{\mathscr{S}}_{t}} \log \left[p\left(x_{t}, y_{t} \mid \boldsymbol{\theta}\right)\right] p\left(x_{t} \mid y_{t}, \theta_{0}\right) d x_{t} .
$$

For the SISO case, the quotient $I_{t}^{(1)} / I_{t}^{(0)}$ can be expressed as:

$$
I_{t}^{(1)} / I_{t}^{(0)}=-\left.\frac{\sqrt{2}}{\sqrt{\pi}} \frac{e^{-x^{2} / 2}}{\operatorname{erf}(x / \sqrt{2})}\right|_{\tilde{\mathscr{t}_{t}}} .
$$

Thus, we can write the FIM as

$$
\begin{aligned}
\mathscr{I}_{F} & =\mathbb{E}\left\{\left.\left.\frac{\partial l(\theta)}{\partial \theta}\right|_{\theta_{0}} \frac{\partial l(\theta)}{\partial \theta^{T}}\right|_{\theta_{0}}\right\} \\
& =\frac{2}{\pi \sigma^{2}} \mathbb{E}\left\{\left(\frac{\left.e^{-x^{2} / 2}\right|_{\tilde{\mathscr{t}}}}{\left.\operatorname{erf}(x / \sqrt{2})\right|_{\tilde{\mathscr{F}_{t}}}}\right)^{2} \varphi_{t} \varphi_{t}^{T}\right\} .
\end{aligned}
$$

Since $x_{t}$ and $\varphi_{t}$ are asymptotically jointly stationary processes, asymptotically uncorrelated (for sufficiently large lags), by the Birkhoff-Khinchin ergodic theorem [25, Lemma B.1], we can write (28) as:

$$
\mathscr{I}_{F}=\lim _{N \rightarrow \infty} \frac{2}{\pi \sigma^{2} N} \sum_{t=1}^{N}\left(\frac{\left.e^{-x^{2} / 2}\right|_{\tilde{\mathscr{T}}}}{\left.\operatorname{erf}(x / \sqrt{2})\right|_{\tilde{\mathscr{I}}}}\right)^{2} \varphi_{t} \varphi_{t}^{T} .
$$

Expression (29) can be estimated by taking a large but finite $N$, obtaining the expression in (22).

Remark 5: Notice that a more general case can be developed considering incomplete data. In particular, missing data is a common type of incomplete data, and to calculate the FIM we still can use the Fisher identity, but we need to consider that the expectations in (9) are now taken with respect to a different set of observations. However, one important assumption to consider is that the missing data mechanism is somehow independent of the coarsening process, see e.g. [3]. 

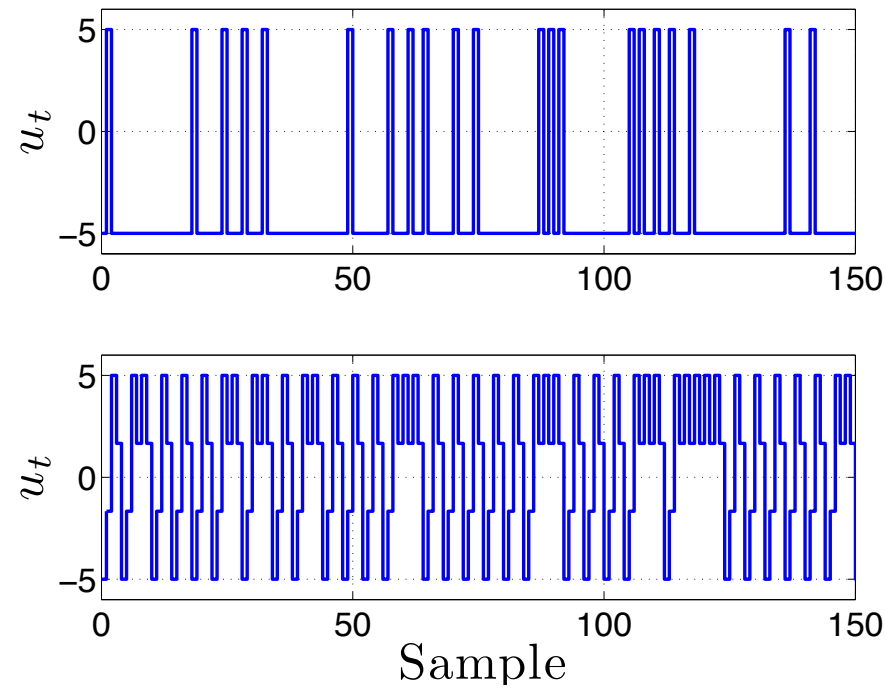

Fig. 4. Input realizations for memory length 2. Top: $c=2$. Bottom: $c=4$.

\section{EXAMPLE}

Here, we consider the following SISO FIR system:

$$
\begin{aligned}
& x_{t}=\varphi_{t}^{T} \theta+w_{t}, \\
& y_{t}=\mathscr{Q}\left[x_{t}\right],
\end{aligned}
$$

where $\mathscr{Q}$ is a 2 -bit quantizer, $\varphi_{t}^{T}=\left[\begin{array}{ll}u_{t-1} & u_{t-2}\end{array}\right], \quad \theta=$ $\left[\begin{array}{ll}0.4 & 1\end{array}\right]^{T}$, and the noise variance is $\sigma^{2}=1$. The extreme values for $\mathscr{Q}$ are -7.5 and 7.5 , with equally spaced output values on $[-7.5,7.5]$.

We use the method presented in Section IV to design an experiment for identifying $\theta$ in (30). For this purpose, we consider an input with maximum amplitude 5 , and different scenarios, composed by memory length in $\{2,3\}$, and $c \in$ $\{2,4\}$. We solve (16) for $h(\cdot)=\operatorname{tr}\left\{W(\cdot)^{-1}\right\}$, where

$$
W=\left[\begin{array}{ll}
4 & 3 \\
3 & 4
\end{array}\right]
$$

The approximation of each $\mathscr{I}_{F}^{(i)}$ in (17) is obtained by using (22) with $N_{\mathrm{sim}}=5 \cdot 10^{5}$. The problem is solved in Matlab using the cVx toolbox [4].

Once the problem (16) is solved, we generate an input signal of length $N=10^{6}$ from the optimal pmf by running a Markov chain based on (20). Typical input realizations for memory length 2 are presented in Figure 4. To compare the results, we compute $h\left(\mathscr{I}_{F}\right)$ for each optimal input signal using (22).

The results obtained for the different scenarios are presented in Table I. The results show that the experiment is improved when we increase the number of possible values for the input $c$. However, there is no significant improvement when we increase the memory length of the stationary process, in agreement with Footnote 3 (which says that a memory length equal to $q$ is optimal).

As a benchmark, we generate a binary white noise input of length $N$ and amplitude 5 , and we compute $h\left(\mathscr{I}_{F}\right)$ for this
TABLE I

$h\left(\mathscr{I}_{F}\right)$ FOR DIFFERENT VALUES OF $c$ AND MEMORY LENGTH (OF THE MARKOV CHAIN INPUT).

\begin{tabular}{|c|c|c|}
\hline memory length $\backslash c$ & 2 & 4 \\
\hline 2 & 3.12 & 1.58 \\
\hline 3 & 3.08 & 1.58 \\
\hline
\end{tabular}

input using (22). The value we obtain is $h\left(\mathscr{I}_{F}\right)=3.75$, which is $16.8 \%$ worse than the worst result presented in Table I. Therefore, for this example the proposed input design method outperforms the result obtained with binary white noise.

\section{CONClusions}

We have developed a novel approach to design input signals for quantized systems. The advantage of our method is that it gives a sequence of temporal input signals which can easily be implemented for systems subject to output quantization.

We have found that, in general, there is an improvement when we consider our design compared to a simple binary white noise signal. For a particular example, this improvement is around $16.8 \%$ when compared with the worst experiment obtained by our method. This result can be further improved by increasing the size of the alphabet for the input signal, at the expense of a higher computational cost.

Future research includes robustifying the input design for systems with quantized output against uncertainty in the knowledge of the true system.

\section{REFERENCES}

[1] J. C. Agüero and G. C. Goodwin. Choosing between open- and closedloop experiments in linear system identification. IEEE Transactions on Automatic Control, 52(8):1475-1480, August 2007.

[2] J.C. Agüero, B.I. Godoy, G.C. Goodwin, and T. Wigren. Scenariobased EM algorithm for FIR systems having quantized data. In 15th IFAC SYSID, Saint-Malo, France, 2009.

[3] J.C. Agüero, W. Tang, J.I. Yuz, R. Delgado, and G.C. Goodwin. Dual time-frequency domain system identification. Automatica, 48:30313041, 2012.

[4] S. Boyd and L. Vandenberghe. Convex Optimization. Cambridge University Press, 2003.

[5] M. Casini, A. Garulli, and A. Vicino. Input design for worst-case system identification with uniformly quantized measurements. In 15th IFAC Symposium on System Identification (SYSID), Saint-Malo, France, 2009.

[6] M. Casini, A. Garulli, and A. Vicino. Input design in worst-case system identification using binary sensors. IEEE Transactions on Automatic Control, 56(5):1186-1191, 2011.

[7] M. Casini, A. Garulli, and A. Vicino. Input design in worst-case system identification with quantized measurements. Automatica, 48(5):2297-3007, 2012.

[8] D. R. Cox. Planning of Experiments. Wiley, New York, 1958.

[9] V. V. Fedorov. Theory of Optimal Experiments. Academic Press, New York, 1972.

[10] M. Gevers and L. Ljung. Optimal experiment designs with respect to the intended model application. Automatica, 22(5):543-554, 1986.

[11] B.I. Godoy, G.C. Goodwin, J.C. Agüero, D. Marelli, and T. Wigren. On identification of FIR systems having quantized output data. Automatica, 46(9):1905-1915, 2011.

[12] G. C. Goodwin and R. L. Payne. Dynamic System Identification: Experiment Design and Data Analysis. Academic Press, New York, 1977. 
[13] R.B. Gopaluni, T.B. Schön, and A.G. Wills. Input design for nonlinear stochastic dynamic systems - A particle filter approach. In 18th IFAC World Congress, Milano, Italy, 2011.

[14] H. Hjalmarsson. From experiment design to closed-loop control. Automatica, 41(3):393-438, March 2005.

[15] H. Hjalmarsson and J Mårtensson. Optimal input design for identification of non-linear systems: Learning from the linear case. In Proceedings of the American Control Conference (ACC'07), New York, USA, 2007.

[16] D. B. Johnson. Finding all the elementary circuits of a directed graph. SIAM Journal on Computing, 4(1):77-84, 1975.

[17] O. Kempthorne. Design and Analysis of Experiments. Wiley, New York, 1952.

[18] C. Larsson, H. Hjalmarsson, and C. R. Rojas. On optimal input design for nonlinear systems. In Proceedings of the 49th Conference on Decision and Control (CDC'10), Atlanta, USA, 2010.

[19] D. Marelli, K. You, and M. Fu. Identification of ARMA models using intermittent and quantized output observations. Automatica, 49:360369, 2013.

[20] G.J. Mclachlan and T. Krishnan. The EM algorithm and Extensions. John Wiley \& Sons, Inc., 2nd edition, 2008.

[21] R. K. Mehra. Optimal input signals for parameter estimation in dynamic systems - survey and new results. IEEE Transactions on Automatic Control, 19(6):753-768, December 1974.

[22] C. R. Rojas, J. C. Agüero, J. S. Welsh, G. C. Goodwin, and A. Feuer. Robustness in experiment design. IEEE Transactions on Automatic Control, 57(4):860-874, 2012.

[23] C. R. Rojas, J. S. Welsh, G. C. Goodwin, and A. Feuer. Robust optimal experiment design for system identification. Automatica, 43(6):9931008, 2007.

[24] C.R. Rojas, J.S. Welsh, G.C. Goodwin, and A. Feuer. Robust optimal experiment design for system identification. Automatica, 43(6):993$1008,2007$.

[25] T. Söderström and P. Stoica. System Identification. Prentice Hall, Hertfordshire, United Kingdom, 1989.

[26] R. Tarjan. Depth-first search and linear graph algorithms. SIAM Journal on Computing, 1(2):146-160, 1972.

[27] P. E. Valenzuela, C. R. Rojas, and H. Hjalmarsson. A graph theoretical approach to input design of dynamic systems. Automatica (submitted for publication), 2013.

[28] P. E. Valenzuela, C. R. Rojas, and H. Hjalmarsson. Optimal input design for dynamic systems: a graph theory approach. In Proceedings of the IEEE Conference on Decision and Control (CDC'13), 2013.

[29] T.L. Vincent, C. Novara, K. Hsu, and K. Poola. Input design for structured nonlinear system identification. In 15th IFAC Symposium on System Identification, pages 174-179, Saint-Malo, France, 2009.

[30] L.Y. Wang, G.G. Yin, J-F. Zhang, and Y. Zhao. System Identification with Quantized Observations. Birkhäuser, 2010.

[31] A. Zaman. Stationarity on finite strings and shift register sequences. The Annals of Probability, 11(3):678-684, 1983.

[32] M. Zarrop. Optimal Experiment Design for Dynamic System Identification. Springer, Berlin, 1979. 